\title{
Impact of Prolonged Face Mask Wearing On Tear Break-Up Time and Dry Eye Symptoms in Health Care Professionals
}

\section{Mine Esen Baris ( $\nabla$ mine.baris@yahoo.com )}

Ege Universitesi Tip Fakultesi https://orcid.org/0000-0003-1341-6737

\section{Suzan Guven Yilmaz}

Ege University Faculty of Medicine: Ege Universitesi Tip Fakultesi

Melis Palamar

Ege University Faculty of Medicine: Ege Universitesi Tip Fakultesi

\section{Research Article}

Keywords: dry eye, mask associated dry eye, surgical face mask

Posted Date: September 30th, 2021

DOI: https://doi.org/10.21203/rs.3.rs-886517/v1

License: (c) (1) This work is licensed under a Creative Commons Attribution 4.0 International License.

Read Full License 


\section{Abstract \\ Purpose}

To evaluate the impact of prolonged surgical face mask wearing on dry eye symptoms and tear film break-up time (T-BUT) in health care professionals.

\section{Materials and Methods}

A total of 33 health care professionals were included in this cross sectional, prospective study. T-BUT measurements were performed twice for all participants in the morning $(8 \mathrm{am})$ and in the afternoon (5 $\mathrm{pm}$ ) in addition to a complete ophthalmological examination. The subjects also filled-in the ocular surface disease index (OSDI) questionnaire twice, before and after wearing the face mask, on the same day.

\section{Results}

Sixty six eyes of 33 participants ( 17 female and 16 male) were evaluated. The mean age was $33.6 \pm 7.55$ (24-48) years and mean total duration with mask on between the two evaluations was $514 \pm 12.5$ (495526) minutes. The mean T-BUT was $9.3 \pm 1.0(3-16)$ seconds at 8 am and $8.3 \pm 1.5(3-14)$ seconds at 5 $\mathrm{pm}(\mathrm{p}=0.01)$. The mean OSDI score was $20.1 \pm 8.3(0-68.75)$ at 8 am and $27.4 \pm 10.4(0-81.25)$ at $5 \mathrm{pm}$ $(p<0.01)$.

\section{Conclusion}

Use of a surgical mask for the entire work day was seen to worsen T-BUT and dry eye symptoms in healthy individuals. Ophthalmologists should be aware of possible worsening of condition in patients with dry eye with the prolonged use of face masks and consider modifications if necessary.

\section{Introduction}

Face masks are effective in preventing the spread of viruses. Since the beginning of the COVID-19 pandemic, face masks, along with other measures such as social distancing and hand sanitation, became essential in our daily lives. With the increasing frequency and duration of face mask use, many ophthalmologists and physicians reported an increase in dry eye symptoms associated with face mask usage $[1,2]$. The term "mask associated dry eye (MADE)" was coined to describe this condition [3]. There are many short reports, online surveys and letters on MADE; however, objective studies have not yet been reported. 
In this cross-sectional study, we aimed to evaluate the effect of prolonged face mask wearing in health care professionals on tear film break-up time (T-BUT) and determine its relationship with dry eye symptoms.

\section{Materials And Methods}

Health care professionals, including ophthalmology residents and nurses working in an ophthalmology department, were included in the study. Participants with a medical history of systemic or ocular diseases (including dry eye diagnosis) or undergoing systemic or topical treatments (including lubricants or artificial tears) and contact lens wearers were excluded from the study. Each patient underwent a complete ophthalmological examination including best corrected visual acuity (BCVA), intraocular pressure (IOP) measurement, biomicroscopic anterior segment examination and fundus examination with 90 D lens. BCVA was measured with the Snellen chart. IOP measurements were performed with a Goldmann applanation tonometer at the end of the work day, after all other tests were completed. T-BUT was evaluated in all subjects twice while the surgical masks were on: when they first started wearing the surgical face mask (at $8 \mathrm{am}$ ) and at the end of the work day $(5 \mathrm{pm})$. T-BUT was measured three times in both visits and the mean of these three measurements was taken into consideration. The subjects were asked make a note of the duration they kept their masks on during the work day. All the participants were also asked to fill in the Ocular Surface Disease Index (OSDI) Questionnaire twice, before wearing the mask in the morning and after removing the mask at the end of the work day. Both eyes of all the subjects were included in the statistical analyses.

SPSS v22.0 (SPSS, Chicago, II, USA) was used for statistical analyses and paired samples t test and Mann Whitney $U$ test were used for comparison. An institutional review board approval from Ege University Local Ethics Committee was taken and informed consent was obtained from all participants. This study was conducted in accordance with the tenets of the Declaration of Helsinki.

\section{Results}

Sixty-six eyes of 33 subjects were included in the study. Seventeen (51.5\%) participants were female and $16(48.5 \%)$ were male. Mean age was $33.6 \pm 7.55$ (24-48) years. During the 9-hour period between the two measurements, the mean total duration that the subjects kept their mask on was $514 \pm 12.5$ (495526) minutes. Masks were taken off mostly during lunch and coffee breaks. Mean BCVA was $0.8 \pm 0.16$ (0.7-1) and mean IOP was $14.6 \pm 4.8(11-19) \mathrm{mmHg}$. All subjects had normal fundus examination findings. The mean T-BUT was $9.3 \pm 1.0(3-16)$ seconds at 8 am; this value was significantly reduced to $8.3 \pm 1.5(3-14)$ seconds at $5 \mathrm{pm}(p=0.01$, paired samples $t$ test $)$. The OSDI scores of $19(57.6 \%)$ participants were higher at the end of the day, compared to the morning OSDI scores. The mean OSDI score in the morning was $20.1 \pm 8.3$ (0-68.75), which significantly increased to $27.4 \pm 10.4$ at the end of the work day $(0-81.25)(\mathrm{p}<0.01$, paired sample $t$ test). The worsening of OSDI and T-BUT scores did not show any statistically significant difference between female and male participants $(p>0.05$, Mann Whitney $U$ test). The statistical analysis data are summarized in Table 1. 


\section{Discussion}

Ever since the use of surgical face masks all-day long became essential due to the pandemic and a part of our daily lives, doctors and health care staff all over the world have been complaining of dry eye symptoms. In this study, we aimed to investigate the impact of long-term face mask wearing on T-BUT and dry eye symptoms in health care professionals.

To the best of our knowledge, this is the first study to evaluate the effects of long term face mask wearing on OSDI score and T-BUT. Our findings indicate that both OSDI scores and T-BUT significantly worsened at the end of the day, compared to the morning hours. The reason might be increased evaporation caused by air flow around the eye, especially if the mask is not tight enough. Patients with dry eye syndrome, however, are also known to report discomfort that is more prominent in the evening hours, compared to mornings. In a study with self-made tear strip meniscometry in healthy eyes, Ayaki et al reported that tear meniscus measurements were the highest in the morning and gradually decreased in the evening hours [4]. Therefore, in the current study, to firmly establish that wearing the face mask was the reason for differences in T-BUT and OSDI scores, a matched control group of participants who did not use a surgical face mask throughout the day was necessary. Unfortunately, due to precautions needed during the ongoing COVID-19 pandemic, such a control group cannot be incorporated in the study in the near future and until it is safe to do so.

Giannacare et al. reported in their online survey study with 107 healthy subjects that $10.3 \%$ of the participants described the development or worsening in dry eye symptoms [5]. Boccardo reported in a survey study with 3605 subjects that $26.9 \%$ of participants with a prior history of dry eye experienced worsening in symptoms while a total of $18.3 \%$ of the participants experienced MADE [1]. In the current study, we observed a higher prevalence in the worsening of symptoms (57.6\%). This can be due to the inclusion of a more homogenous study population (health care professionals) while the other published survey studies included a more heterogeneous group of people from different professions. All of the participants in the current study were working in a hospital environment with air conditioners, spending a considerable time in front of computers and were strictly adhering to the rules of face mask wearing for long hours. Although 21 (63.7\%) of the participants in the current study had higher than normal morning OSDI scores (>15), none of the participants had previous dry eye syndrome diagnoses or were using artificial tears. Despite the presence of some symptoms of dry eye, $12(57.1 \%)$ of the subjects had normal T-BUT values (> 10 seconds).

Dry eye disease is known to have a higher prevalence amongst women, compared to men [6]. In the present study, dry eye symptoms and T-BUT values did not show any significant difference between male and female participants. This could be related to the identical environmental factors that health care professionals of both genders were subject to. Moreover, all participants were young with a mean age of 34 years and none of them reported any menopausal or hormonal effects [7]. It can also be suggested that the lack of any systemic or dermatologic diseases in either male or female participants might have affected the study outcomes [8-10]. 
Similar to the use of facial masks, the use continuous positive airway pressure (CPAP) treatment is known to be associated with ocular surface complications related to the increased air flow around the eye [11]. Especially, misplacement of the CPAP mask during sleep was reported to cause more dry eye symptoms in the eye for which the air flow was more prominent [11]. Surgical face masks, on the other hand, were not considered as an additional risk factor for dry eye until the COVID-19 pandemic. Now that surgical face masks are an essential part of our lives and also our health, it is important to use them comfortably for long durations. Since individuals with no history of dry eye have been reporting symptoms with long hours of face mask use, ophthalmologists should be aware of this and should be willing to offer solutions. Lubricants might be beneficial for people with new onset symptoms suggesting MADE. Patients with moderate to severe dry eye might require modifications in their treatment regime.

In the current study, the relationship between use of face masks and Meibomian gland dysfunction was not evaluated. However, anecdotal evidence, including observations in our daily practice suggest a higher prevalence of chalazion than usual. Silkiss et al. reported an increased incidence of chalazion in a retrospective study and implicated increased air flow around the eye and the eyelids with the use of face masks [12]. Further studies are needed to assess the effect of long term surgical mask use on Meibomian gland functions. Ameliorating the symptoms of dry eye might even increase compliance to the use of face mask, prevent the spread of the virus and help protect individuals from COVID-19.

\section{Declarations}

\section{Acknowledgements}

None

Funding: This study was not funded by any source.

Conflict of interests: The authors declare that there is no conflicting interests.

Availability of data and material: Not applicable

Code availability: Not applicable

Authors' contributions: Mine Esen Baris: Data acquisition, writing of the manuscript; Suzan Guven Yilmaz: Data analysis; Melis Palamar: Design of the study, critical editing of the manuscript.

Ethics approval: Ethics approval was gathered from Ege University Clinical Studies Ethics Committee.

\section{References}

1. Boccardo L. Self-reported symptoms of mask-associated dry eye: A survey study of 3,605 people. Contact Lens and Anterior Eye. Cont Lens Anterior Eye. 2021 Jan 20;101408. doi: 10.1016/j.clae.2021.01.003. 
2. Pandey SK, Sharma V. Mask-associated dry eye disease and dry eye due to prolonged screen time: Are we heading towards a new dry eye epidemic during the COVID-19 era? Indian J Ophthalmol 2021 Feb;69(2):448-449. doi: 10.4103/ijo.IJO_3250_20.

3. White DE. BLOG: MADE: a new coronavirus-associated eye disease. 2020. https://www.healio.com/news/ophthalmology/20200622/blog-a-new-coronavirusasso ciated-eyedisease

4. Ayaki $\mathrm{M}$, Tachi $\mathrm{N}$, Hashimato $\mathrm{Y}$ et al.Diurnal variation of human tear meniscus volume measured with tear strip meniscometry self-examination. PLoS One 2019 Apr 23;14(4):e0215922. doi: 10.1371/journal.pone.0215922.

5. Giannacare G, Vaccaro S, Mancini A et al. Dry eye in the COVID-19 era: how the measures for controlling pandemic might harm ocular surface. Graefe's Archive for Clinical and Experimental Ophthalmology. 19 June. Graefes Arch Clin Exp Ophthalmol. 2020 Nov;258(11):25672568. https://doi.org/10.1007/s00417-020-04808-3

6. Stapleton F, Alves M, Bunya VY, et al. TFOS DEWS II epidemiology report. Ocul Surf 2017;15:334-65. https://doi.org/10.1016/j.jtos.2017.05.003.

7. Garcia-Alfaro P, Garcia S, Rodriguez I et al. Dry eye disease symptoms and quality of life in perimenopausal and postmenopausal women. Climacteric. 2020 Dec 7;1-11. doi:

10.1080/13697137.2020.1849087

8. Palamar M, Degirmenci C, Ertam I et al. Evaluation of dry eye and meibomian gland dysfunction with meibography in patients with rosacea Cornea. 2015 May;34(5):497-9.

9. Palamar M, Kiyat P, Ertam I et al. Evaluation of dry eye and meibomian gland dysfunction with meibography in vitiligo. Eye (Lond). 2017;31(7):1074-1077.

10. Adiguzel S, Palamar M, Yargucu F et. al. Evaluation of Ocular Surface and Meibomian Glands in Patients With Scleroderma. Cornea 2020; Oct 19. doi: 10.1097/IC0.0000000000002551.

11. Hayirci E, Yagci A, Palamar M et al. The effect of continuous positive airway pressure treatment for obstructive sleep apnea syndrome on the ocular surface. Cornea 2012 Jun;31(6):604-8.

12. Silkiss RZ, Paap MK, Ugradar S. Increased incidence of chalazion associated with face mask wear during the COVID-19 pandemic. Am J Ophthalmol Case Rep. 2021 Jun;22:101032. doi: 10.1016/j.ajoc.2021.101032. Epub 2021 Feb 9.

\section{Tables}


Table 1

Tear film break-up time and Ocular Surface Disese Index (OSDI) scores in the morning and evening hours.

\begin{tabular}{|llll|}
\hline & $\mathbf{0 8 . 0 0}$ am & $\mathbf{1 7 . 0 0 ~ p m}$ & P value \\
\hline OSDI score & $20.1 \pm 8.3(0-68.75)$ & $27.4 \pm 10.4(0-81.25)$ & $<0.01 *$ \\
\hline T-BUT & $9.3 \pm 1.0(3-16)$ & $8.3 \pm 1.5(3-14)$ & $0.01 *$ \\
\hline $\begin{array}{l}\text { OSDI: Ocular Surface Disease Index; T-BUT: Tear film break-up time } \\
\text { *Paired sample t test }\end{array}$ & & \\
\hline
\end{tabular}

